\title{
Cost-utility analysis of telemonitoring versus conventional hospital-based follow-up of patients with pacemakers. The NORDLAND randomized clinical trial.
}

Lopez-Villegas A, Catalan-Matamoros D, Peiro S, Tore Lappegard K, Lopez-Liria R.

\section{DO FILE (STATA)}

* PATIENTS' CLINICAL CHARACTERISTICS AT BASELINE (TABLE 1)

** "summarize" command (for describing) and "ttest" command (for differences

between groups) for quantitative variables, were used, and "tab" command with chi square or Fisher's exact test for qualitative variables.

** Age and sex.

by ARM_N, sort: summarize AGE, detail

ttest AGE, by (ARM_N)

tab SEX ARM_N, row col chi exact

prtest SEX, by (ARM_N)

** Pacing indication, symptoms and stimulation.

tab ORIGIN ARM_N, row col chi exact

tab INDICATION ARM_N, row col chi exact

tab SYMPTOMS ARM_N, row col chi exact

tab STIMULATION ARM_N, row col chi exact

* Comorbidities: high blood pressure, coronary heart disease, diabetes, tachyarrhythmia, obesity (BMI>30), other, none.

tab C_HBP ARM_N, row col chi exact

tab C_CHD ARM_N, row col chi exact

tab C_TACHYARRHYT ARM_N, row col chi exact

tab C_DISLYP ARM_N, row col chi exact

tab C_DM ARM_N, row col chi exact

tab C OBESI ARM N, row col chi exact

tab C_OTHER2 ARM_N, row col chi exact

tab C_NONE ARM_N, row col chi exact

** Treatment (basal) : antiplatelet agents, oral anticoagulants, anti-arrhythmic drugs, anti-hypertensive drugs.

tab TR_PLAQ ARM_N, row col chi exact

tab TR_OAC ARM_N, row col chi exact

tab TR_ARRIT ARM_N, row col chi exact

tab TR_ANTIHT ARM_N, row col chi exact

** EQ5D basal (month 0) and at first month.

by ARM_N, sort: summarize EQ5D_MONTH_0, detail

ttest EQ5D_MONTH_0, by (ARM_N)

by ARM_N, sort: summarize EQ5D_MONTH_1, detai1

ttest EQ5D_MONTH_1, by (ARM_N) 
* COST INPUTS, COSTS PER PATIENT YEAR, AND QUALITY OF LIFE OUTCOMES (TABLE 2)

** "ttest" command for differences between groups was used.

* * UTILIZATION

** In-office visits, patient/year

ttest n_HOSP_TRANSM, by (ARM_N)

** Pacemaker transmission, patient/year

ttest t_TRANSM, by (ARM_N)

* Physician time, min/patient

ttest PHYS_MIN, by (ARM_N)

* Hospitalization days

ttest HOSP_DAYS, by (ARM_N)

** Distance home/hosp (Km)

ttest DISTANCE, by (ARM_N)

** Patients' time (travel \& visits)

ttest PATIENT_TIME, by (ARM_N)

** NHS COSTS

* Physician costs

ttest COST_DR, by (ARM_N)

* Consultation room costs

ttest COST_ROOM, by (ARM_N)

** Hospitalization costs

ttest COST_HOSP, by (ARM_N)

** Ambulance transport costs

ttest COST_AMBUL, by (ARM_N)

* Total NHS costs

generate tCOSTS_NHS $=$ COST_DR + COST_ROOM + COST_HOSP + COST_AMBUL

label variable tCOSTS_NHS "Total costs NHS perspective"

format \%9. 2 tCOSTS_NHS

ttest tCOSTS_NHS, by (ARM_N)

** PATIENT COSTS

* * Patient travel costs

ttest COST_P_TRAVEL, by (ARM_N)

* Patient travel+waiting costs

ttest COST_P_TRAV_WAIT, by (ARM_N)

** other transport costs

ttest COST_P_OTH_TRAV, by (ARM_N)

* Accompanying person costs

ttest COST_P_ACOMP, by (ARM_N)

ttest HOSP_CONS, by (ARM_N)

* Total patient costs

generate tCOST_PATIENT $=$ COST_P_TRAV_WAIT + COST_P_OTH_TRAV + COST_P_ACOMP

label variable tCOST_PATIENT "Total patient\&family costs" 
format \%9.2f tCOST_PATIENT

ttest tCOST_PATIENT, by (ARM_N)

** TOTAL (NHS + PATIENT\&FAMILY) COSTS

generate tCOSTS $=$ tCOSTS_NHS + tCOST_PATIENT

label variable tCOSTS "Total costs (NHS+Patients\&family)"

ttest tCOSTS, by (ARM_N)

* * OUTCOMES

** Hospitalizations and deaths

by ARM_N, sort: ci HOSPITALIZ, binomial total

prtest HOSPITALIZ, by (ARM_N)

by ARM_N, sort: ci DEATH, binomial total

prtest DEATH, by (ARM_N)

** Quality of life at 6 and 12 months

ttest EQ5D_MONTH_6, by (ARM_N)

by ARM_N, sort: ci HOSPITALIZ, binomial total

ttest EQ5D_MONTH_12, by (ARM_N)

** Generating and analyzing QALYs

* * Generating QALY1 as lineal interpolation between EQ5D weights at month 1 and 6 , between month 6 and 12, and between the two resultant values.

generate QALY_1 $=((($ EQ5D_MONTH_1 + EQ5D_MONTH_6 $) / 2)+(($ EQ5D_MONTH_6 + EQ5D_MONTH_12)/2)) /2

label variable QALY_1 "QALYs"

format \%9. 4f QALY_1

ttest QALY_1, by (ARM_N)

* COSTS PER PATIENT/YEAR AND COST-UTILITY ANALYSIS (TABLE 3)

** Generating cost per QALY variables and testing differences.

generate NHSCOST_Q1 $=$ tCOSTS_NHS/QALY_1

replace NHSCOST_Q1 $=0$ if NHSCOST_Q1==.

generate TCOST_Q1 = tCOSTS/QALY_1

replace TCOST $Q 1=0$ if TCOST $Q 1==$.

label variable NHSCOST_Q1 "Cost/QALY NHS perspective"

format \%9. 2 f NHSCOST_Q1

label variable TCOST_Q1 "Total Cost/QALY"

format \%9.2f TCOST_Q1

ttest NHSCOST_Q1, by (ARM_N)

ttest TCOST_Q1, by (ARM_N)

** Cost-utility analysis (total NHS costs and total costs) using heabs stata command (with a WTP threshold of 30000 and 50000 euros).

heabs tCOSTS_NHS QALY_1, res (bene) int (ARM_N) w2p (30000)

heabs tCOSTS_NHS QALY_1, res (bene) int (ARM_N) w2p (50000)

heabs tCOSTS QALY_1, res (bene) int (ARM_N) w2p (30000) 
heabs tCOSTS QALY_1, res (bene) int (ARM_N) w2p (50000)

* The probabilistic analysis was carried out with the HDS online calculator (http://www.healthstrategy.com/evpiinb/evpiinb01.htm)

* SUPPLEMENTAL ANALYSIS (TABLE S2.2. COSTS PER PATIENT-YEAR EXCLUDING HOSPITALIZATION DAYS COSTS)

generate tCOST_NHS_W = tCOSTS_NHS - COST_HOSP

generate tCOST_W $=$ tCOSTS - COST_HOSP

label variable tCOST_NHS_W "Total costs NHS perspective W/0 hospitalization costs" label variable tCOST_W "Total costs W/0 hospitalization costs"

format \%9.2f tCOST_NHS_W

format \%9. 2 f tCOST_W

ttest tCOST_NHS_W, by (ARM_N)

ttest tCOST_W, by (ARM_N)

** Generating cost (w/O hospitalization days) per QALY variables.

generate NHSCOSTW_Q1 = tCOST_NHS_W/QALY_1

replace NHSCOSTW_Q1 $=0$ if NHSCOSTW_Q1==.

generate TCOSTW_Q1 $=$ tCOST_W/QALY_1

replace TCOSTW_Q1=0 if TCOSTW_Q1==.

label variable NHSCOSTW_Q1 "Total costs/QALY NHS perspective W/0 hospitalization costs"

labe1 variable TCOSTW_Q1 "Total costs/QALY W/0 hospitalization costs"

format \%9. 2 f TCOSTW_Q1

format \%9. 2f NHSCOSTW_Q1

ttest NHSCOSTW_Q1, by (ARM_N)

ttest TCOSTW_Q1, by (ARM_N)

* SUPPLEMENTAL ANALYSIS (TABLE S2 .3. MEAN COSTS PER PATIENT-YEAR AND COST-UTILITY ANALYSIS, WITHOUT HOSPITALIZATION)

heabs tCOST_NHS_W QALY_1, res (bene) int (ARM_N) w2p (30000)

heabs tCOST_NHS_W QALY_1, res (bene) int (ARM_N) w2p (50000)

heabs tCOST_W QALY_1, res (bene) int (ARM_N) w2p (30000)

heabs tCOST_W QALY_1, res (bene) int (ARM_N) w2p (50000)

* SUPPLEMENTAL ANALYSIS (TABLE S3.1. DISTRIBUTION OF THE MOST RELEVANT VARIABLES BY GROUP: MEDIAN, MIN, P25, P75 AND MAX).

** In-office visits, patient/year

by ARM_N, sort: summarize n_HOSP_TRANSM, detail

** Pacemaker transmission, patient/year

by ARM_N, sort: summarize t_TRANSM, detail

** Physician time, min/patient 
by ARM_N, sort: summarize PHYS_MIN, detail

** Hospitalization days

by ARM_N, sort: summarize HOSP_DAYS, detail

** Distance home/hosp (Km)

by ARM_N, sort: summarize DISTANCE, detail

** Patients' time (travel \& visits)

by ARM_N, sort: summarize PATIENT_TIME, detail

* Physician costs

by ARM_N, sort: summarize COST_DR, detail

** Consultation room costs

by ARM_N, sort: summarize COST_ROOM, detail

* Hospitalization days costs

by ARM_N, sort: summarize COST_HOSP, detail

** Ambulance transport costs

by ARM_N, sort: summarize COST_AMBUL, detail

** Total NHS costs

by ARM_N, sort: summarize tCOSTS_NHS, detail

** Patient travel costs

by ARM_N, sort: summarize COST_P_TRAVEL, detai1

by ARM_N, sort: summarize COST_P_TRAV_WAIT, detail

** other transport costs

by ARM_N, sort: summarize COST_P_OTH_TRAV, detail

** Accompanying person costs

by ARM_N, sort: summarize COST_P_ACOMP, detai1

by ARM_N, sort: summarize HOSP_CONS, detail

** Total patient costs

by ARM_N, sort: summarize tCOST_PATIENT, detail

** Total (NHS + patient family) costs

by ARM_N, sort: summarize tCOSTS, detail

** Total NHS costs and total costs $W / O$ hospitalization costs

by ARM_N, sort: summarize tCOST_NHS_W, detail

by ARM_N, sort: summarize tCOST_W, detail

** EQ5D utilities and QALYS

by ARM_N, sort: summarize EQ5D_MONTH_0, detai1

by ARM_N, sort: summarize EQ5D_MONTH_1, detail

by ARM_N, sort: summarize EQ5D_MONTH_6, detai1

by ARM_N, sort: summarize EQ5D_MONTH_12, detai1

by ARM_N, sort: summarize QALY_1, detail

* Cost per QALY

by ARM_N, sort: summarize NHSCOST_Q1, detai1 
by ARM_N, sort: summarize TCOST_Q1, detai1

by ARM_N, sort: summarize NHSCOSTW_Q1, detai1

by ARM_N, sort: summarize TCOSTW_Q1, detai1 
* patientsõ ClinicAl characteristics AT BASEline (TABLE 1)

** Òsummarizeó command (for describing) and òttestó command (for differences between groups) for quantitative variables, were used, and òtabó command with chi square or Fisherõs exact test for qualitative variables.

** Age and sex.

by ARM_N, sort: summarize AGE, detail

test AGE, by (ARM_N)

tab SEX ARM_N, row COl chi exact

prtest SEX, by (ARM_N)

** Pacing indication, symptoms and stimulation.

tab ORIGIN ARM_N, row col chi exact

tab INDICATION ARM_N, row col chi exact

tab SYMPTOMS ARM_N, row col chi exact

tab STIMULATION ARM_N, row col chi exact

* Comorbidities: high blood pressure, coronary heart disease, diabetes, tachyarrhythmia, obesity (BMI>30), other, none.

tab C_HBP ARM_N, row col chi exact

tab C_CHD ARM_N, row col chi exact

tab C_TACHYARRHYT ARM_N, row col chi exact

tab C_DISLYP ARM_N, row col chi exact

tab C_DM ARM_N, row col chi exact

tab C_OBESI ARM_N, row col chi exact

tab C_OTHER2 ARM_N, row col chi exact

tab C_NONE ARM_N, row col chi exact

** Treatment (basal): antiplatelet agents, oral anticoagulants, antiarrhythmic drugs, anti-hypertensive drugs.

tab TR_PLAQ ARM_N, row COl chi exact

tab TR_OAC ARM_N, row col chi exact

tab TR_ARRIT ARM_N, row COl chi exact

tab TR_ANTIHT ARM_N, row col chi exact

** EQ5D basal (month 0) and at first month.

by ARM_N, sort: summarize EQ5D_MONTH_0, detail

ttest EQ5D_MONTH_0, by (ARM_N)

by ARM_N, sort: summarize EQ5D_MONTH_1, detail

ttest EQ5D_MONTH_1, by (ARM_N)

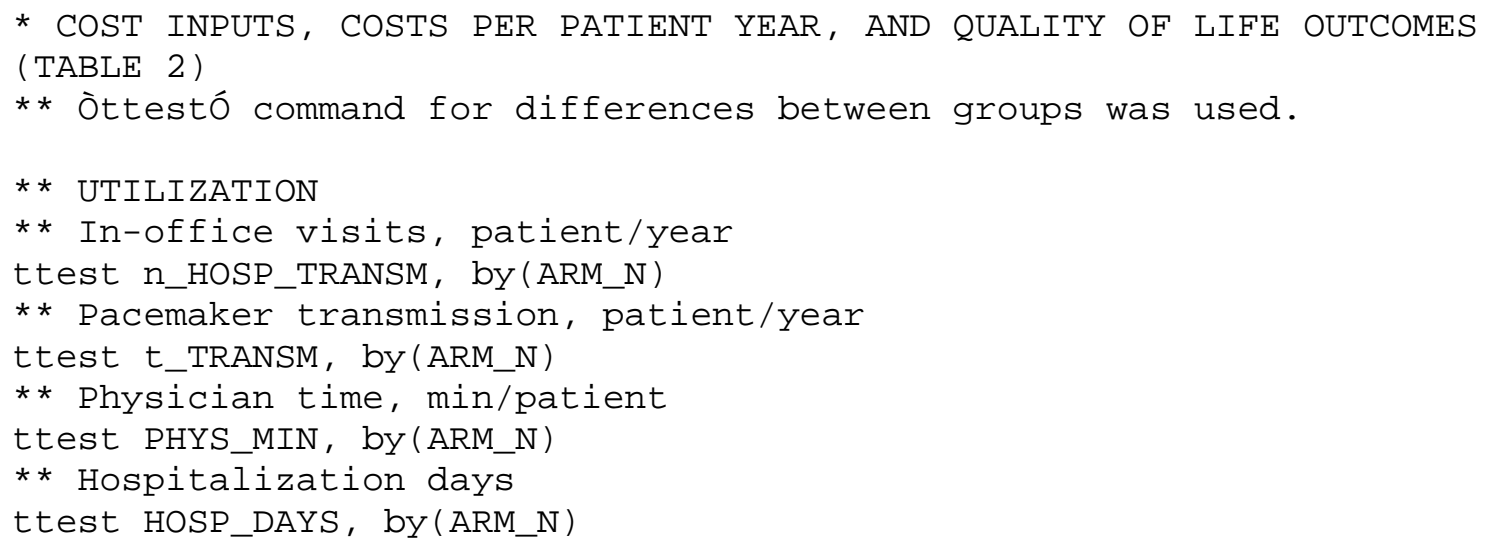




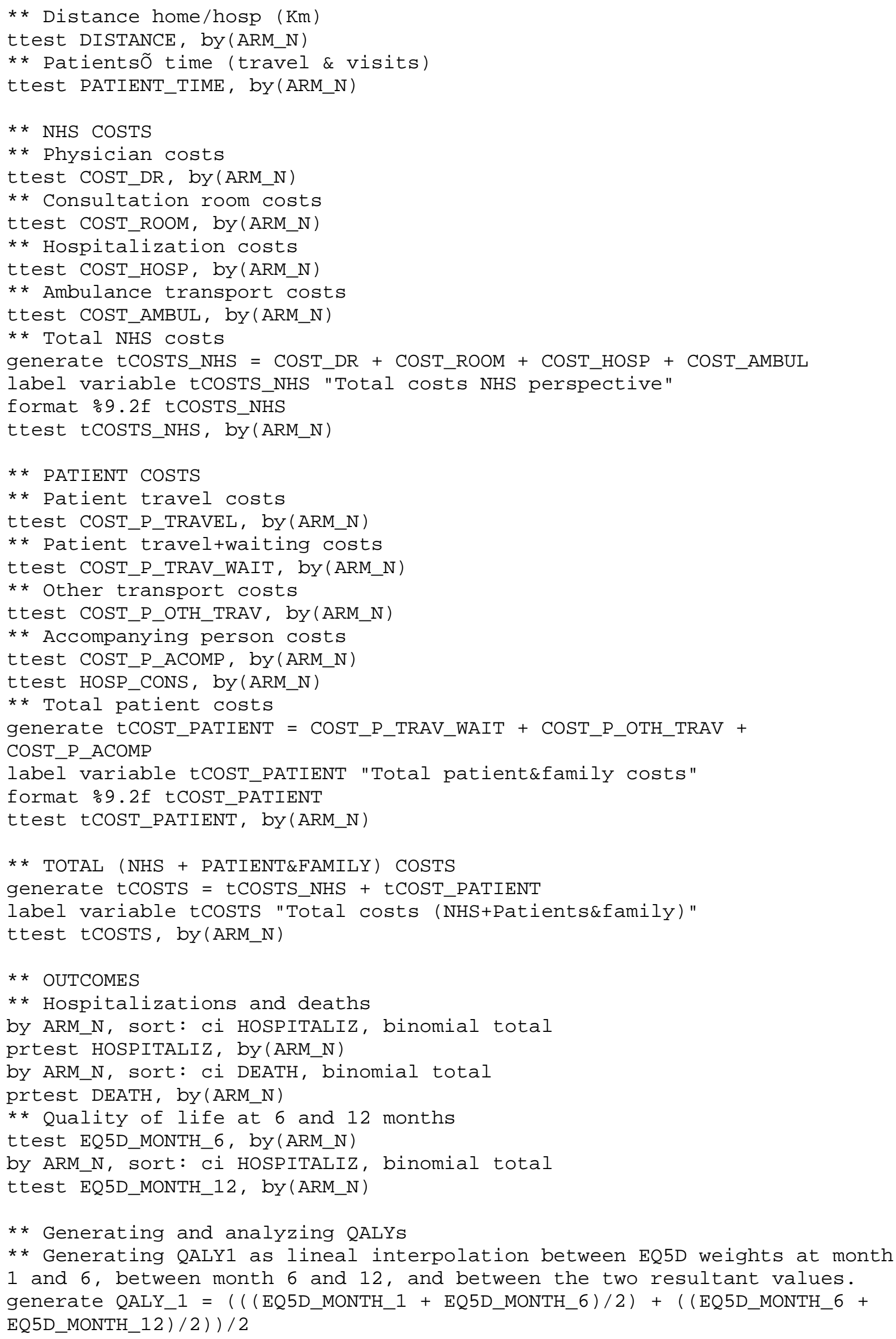


label variable QALY_1 "QALYs"

format $\div 9.4 \mathrm{f}$ QALY_1

test QALY_1, by (ARM_N)

* costs per patient/year And costĐutility anAlysis (TABle 3)

** Generating cost per QALY variables and testing differences.

generate NHSCOST_Q1 = tCOSTS_NHS/QALY_1

replace NHSCOST_Q1=0 if NHSCOST_Q1==.

generate TCOST_Q1 = tCOSTS/QALY_1

replace TCOST_Q1 $=0$ if TCOST_Q1==.

label variable NHSCOST_Q1 "Cost/QALY NHS perspective"

format \%9.2f NHSCOST_Q1

label variable TCOST_Q1 "Total Cost/QALY"

format \%9.2f TCOST_Q1

ttest NHSCOST_Q1, by (ARM_N)

test TCOST_Q1, by (ARM_N)

* Cost-utility analysis (total NHS costs and total costs) using heabs stata command (with a WTP threshold of 30000 and 50000 euros). heabs tCOSTS_NHS QALY_1, res(bene) int (ARM_N) w2p (30000)

heabs tCOSTS_NHS QALY_1, res(bene) int (ARM_N) w2p (50000)

heabs tCOSTS QALY_1, res (bene) int (ARM_N) w2p (30000)

heabs tCOSTS QALY_1, res (bene) int (ARM_N) w2p (50000)

* The probabilistic analysis was carried out with the HDS online calculator (http://www.healthstrategy.com/evpiinb/evpiinb01.htm)

* SUPPlemental ANALysis (TABLE S2.2. COSTS PER PATIENT-yeAR EXCLUDing HOSPITALIZATION DAYS COSTS)

generate tCOST_NHS_W = tCOSTS_NHS - COST_HOSP

generate tCOST_W = tCOSTS - COST_HOSP

label variable tCOST_NHS_W "Total costs NHS perspective W/O

hospitalization costs"

label variable tCosT_W "Total costs W/O hospitalization costs"

format $\% 9.2 \mathrm{f}$ tCOST_NHS_W

format $\% 9.2 \mathrm{f}$ tCOST_W

ttest tCOST_NHS_W, by (ARM_N)

ttest tCOST_W, by (ARM_N)

** Generating cost (w/o hospitalization days) per QALY variables. generate NHSCOSTW_Q1 = tCOST_NHS_W/QALY_1

replace NHSCOSTW_Q1=0 if NHSCOSTW_Q1==.

generate TCOSTW_Q1 = tCOST_W/QALY_1

replace TCOSTW_Q1 $=0$ if TCOSTW_Q1==.

label variable NHSCOSTW_Q1 "Total costs/QALY NHS perspective W/O hospitalization costs"

label variable TCOSTW_Q1 "Total costs/QALY W/O hospitalization costs"

format $\% 9.2 \mathrm{f}$ TCOSTW_Q1

format $\% 9.2 \mathrm{f}$ NHSCOSTW_Q1

ttest NHSCOSTW_Q1, by (ARM_N)

ttest TCOSTW_Q1, by (ARM_N)

* SupPlemental ANALysis (TABle S2.3. MEAN COSTS PER PAtient-yeAR AND COST-UTILITY ANALYSIS, WITHOUT HOSPITALIZATION)

heabs tCOST_NHS_W QALY_1, res (bene) int (ARM_N) w2p (30000)

heabs tCOST_NHS_W QALY_1, res (bene) int (ARM_N) w2p (50000)

heabs tCOST_W QALY_1, res (bene) int (ARM_N) w2p (30000) 
heabs tCOST_W QALY_1, res (bene) int (ARM_N) w2p (50000)

* SUPPlemental ANALysis (TABle S3.1. DistRibution OF the MOST RELEVANT VARIABLES BY GROUP: MEDIAN, MIN, P25, P75 AND MAX).

** In-office visits, patient/year

by ARM_N, sort: summarize n_HOSP_TRANSM, detail

** Pacemaker transmission, patient/year

by ARM_N, sort: summarize t_TRANSM, detail

* * Physician time, min/patient

by ARM_N, sort: summarize PHYS_MIN, detail

* Hospitalization days

by ARM_N, sort: summarize HOSP_DAYS, detail

** Distance home/hosp ( $\mathrm{Km})$

by ARM_N, sort: summarize DISTANCE, detail

** Patientsõ time (travel \& visits)

by ARM_N, sort: summarize PATIENT_TIME, detail

** Physician costs

by ARM_N, sort: summarize COST_DR, detail

* *onsultation room costs

by ARM_N, sort: summarize COST_ROOM, detail

** Hospitalization days costs

by ARM_N, sort: summarize COST_HOSP, detail

* Ambulance transport costs

by ARM_N, sort: summarize COST_AMBUL, detail

* Total NHS costs

by ARM_N, sort: summarize tCOSTS_NHS, detail

* Patient travel costs

by ARM_N, sort: summarize COST_P_TRAVEL, detail

by ARM_N, sort: summarize COST_P_TRAV_WAIT, detail

** Other transport costs

by ARM_N, sort: summarize COST_P_OTH_TRAV, detail

** Accompanying person costs

by ARM_N, sort: summarize COST_P_ACOMP, detail

by ARM_N, sort: summarize HOSP_CONS, detail

** Total patient costs

by ARM_N, sort: summarize tCOST_PATIENT, detail

* Total (NHS + patient family) costs

by ARM_N, sort: summarize tCOSTS, detail

* Total NHS costs and total costs W/O hospitalization costs

by ARM_N, sort: summarize tCOST_NHS_W, detail

by ARM_N, sort: summarize tCOST_W, detail

** EQ5D utilities and QALYs

by ARM_N, sort: summarize EQ5D_MONTH_0, detail

by ARM_N, sort: summarize EQ5D_MONTH_1, detail

by ARM_N, sort: summarize EQ5D_MONTH_6, detail

by ARM_N, sort: summarize EQ5D_MONTH_12, detail

by ARM_N, sort: summarize QALY_1, detail

* Cost per QALY

by ARM_N, sort: summarize NHSCOST_Q1, detail

by ARM_N, sort: summarize TCOST_Q1, detail 
by ARM_N, sort: summarize NHSCOSTW_Q1, detail

by ARM_N, sort: summarize TCOSTW_Q1, detail 